\title{
MOLECULAR RECOGNITION OF SULFAQUINOXALINE AND SULFAPYRIDINE WITH MOLECULARLY IMPRINTED POLYMER
}

\author{
T.C. CHENG ${ }^{a}$, Y.T. HUANG ${ }^{b}$, C.Y.CHANG ${ }^{a}, K . S . Y A O^{a}$, C.C. HWANG ${ }^{a^{*}}$ \\ ${ }^{a}$ Department of Life Science, \\ Mingdao University, Peetow, ChangHua, 52345, Taiwan \\ ${ }^{b}$ Institute of Bioinformatics and Structural Biology, \\ National Tsing Hua University, Hsinchu,Taiwan \\ (Received: April 15, 2008 -Accepted: November 7, 2008)
}

\begin{abstract}
The selective separation of sulfapyridine (SPD) from sulfaquinoxaline (SQX) is investigated by applying high performance liquid chromatography (HPLC) with molecularly imprinted polymer (MIP) as the stationary phase. Herein we report the synthesis of a molecularly imprinting polymer, SPD-MIP, by free radical polymerization process. Because of this powerful method synthetic polymers with specific binding sites to template molecule are provided. In addition, the separation performances were represented by using buffer/acetonitrile $(3 / 2, \mathrm{v} / \mathrm{v})$ as mobile phase under $272 \mathrm{~nm}$ UV detection. In order to compare the chromatographic data from the stationary phase, capacity factor $\left(k^{\prime}\right)$ and separation factors $(\alpha)$ were given. The value of $2.71(\alpha)$ revealed that the MIP was able to recognize structurally subtle differences from the template molecule. Our results are discussed with regard to the amount of template, the composition of the chromatographic mobile phase and adsorption capacity.
\end{abstract}

Keywords: selective separation, sulfapyridine (SPD), sulfaquinoxaline (SQX), high performance liquid chromatography (HPLC), molecularly imprinted polymer (MIP)

\section{INTRODUCTION}

Determination of sulfonamides residues in food, especially in animal liver and kidney or milk, has received much attention in recent years. More than ten different kinds of sulfonamides are known to be used for domestic animals in Taiwan. Therefore, inspection of the residual sulfonamides is considered to be the most important duty for public health agencies. For years there has been a continuous interest for the development of analytical methods for the determination of sulfonamides in varying samples. The current methods for sulfonamides determination have been analyzed successfully by the liquid chromatography (LC) with ultra-violet detection ${ }^{1-4}$, fluorescence detection ${ }^{5,6}$, mass spectrometerry ${ }^{7,8}$ or other $^{9}$. These studies have been applied to pharmaceutical assays as well as in food sample. However, all of these methods involve tedious analytical procedures such as extraction complicated steps that are time consuming and give low resolution; sometimes recoveries are low and variable or must be expensive reagents and sophisticated instruments.

The aim of this work is to develop a simple, selective and more sensitive method for the identification and quantification of the sulfonamides (SQX and SPD, Table 1) by using molecular imprinting technique. In the molecular imprinting process by which functional and cross-linking monomers are copolymerized in the presence of a molecular template ${ }^{10-12}$. After removal of the templates, polymers generate selective binding sites which are complementary to the template. Therefore, MIP is able to rebind the same template or its analogue. Besides, MIP can offer potential advantages in many fields, such as drug separation in analytical chemistry ${ }^{13-15}$ sensitive determination used as sensors ${ }^{16-18}$ and extraction..$^{19-21}$ Of the various application areas of MIPs, separations are by far the broadest and most rapidly expanding.

Table 1.- Sulfonamide Structures, CAS numbers and molecular weights under this study.

\begin{tabular}{|c|c|c|}
\hline Structure & Name & $\begin{array}{c}\text { CAS number } \\
\text { (Molecular } \\
\text { weight) }\end{array}$ \\
\hline & $\begin{array}{c}\text { Sulfaquinoxaline } \\
\text { (SQX) }\end{array}$ & $\begin{array}{c}59-40-45 \\
(300.2)\end{array}$ \\
\hline
\end{tabular}

In our previous studies, we described the preparation of a stationary phase of HPLC based on ketoprofen-imprinted polymer ${ }^{22}$. This method proved to be fast and reproducible, showing linearity over a broad range of sample concentration. To further increase the utility of this technique, we have prepared sulfapyridine-imprinted polymer with methacrylic acid as the functional monomer molecule. The result polymers were packed in an analytical column and evaluated as the stationary phase of HPLC. Liquid chromatographic conditions (mobile phase composition, sample loading, flow rate) were altered in order to achieve the highest resolution. The MIP was successfully applied to the direct detection of SQX and SPD in analysis.

\section{EXPERIMENTAL}

\section{Material}

Sulfapyridine (SPD), sulfaquinoxaline sodium (SQX) were purchased from Sigma (St. Louis, MO, USA). Ethylene glycol dimethacrylate (EGDMA, 98\%) and methacrylic acid (MAA, 99\%) which were distilled to remove the inhibitors in previous using were obtained from Merck (Germany). Acetronitrile, acetone, ethanol, methanol, sodium phosphate and phosphoric acid were purchased from TEDIA (Fairfield, OH, USA). These were all HPLC grade.

Chloroform and acetic acid were from R.D.H. (GC grade) while 2.2'-Azobisisobutyronitrile (AIBN) was obtained from TCI (Tokyo, Japan). Water was double de-ionized.

\section{Preparation of the molecularly imprinted polymer}

A conventional imprinted polymer with SPD as the template were prepared, referred as SPD-MIP. In a conical Erlenmeyer flask with a screw top, SPD (template, $1 \mathrm{~mol} \%$ ) and AIBN (initiator, $0.56 \mathrm{mmol}$ ) were dissolved in acetone $(10 \mathrm{~mL})$ and then EGDMA $(93 \mathrm{~mol} \%)$, MAA $(6 \mathrm{~mol} \%)$ were added. The flask was placed in an ultrasonic water bath until clear solutions were obtained, then cooled on ice and the solutions sparged with nitrogen. The flask was placed under a UV-lamp $(365 \mathrm{~nm})$ at $4 \square$ for $2 \mathrm{~h}$. The resulting polymers were dried in a vacuum oven for $24 \mathrm{~h}$ at room temperature. The hard polymers were ground in a laboratory mortar grinder.

The particles were sieved to collect particles $25-45 \mu \mathrm{m}$ in size. Grinding and sieving were repeated until all material passed the $45 \mu \mathrm{m}$ sieve. Nonimprinted reference polymers were prepared using the same conditions with the exception that template was omitted. The particles were suspended in methanol $(30 \mathrm{~mL})$ by sonication for $3 \mathrm{~min}$, placed in a slurry reservoir, and was then packed in a stainless steel column $(150 \times 4.6 \mathrm{~mm}$ I.D. $)$ using an air-driven fluid pump. The particle weight in each column was approximately $3.32 \mathrm{~g}$. The packing was carried out under a pressure of $300 \mathrm{bar}$ with acetone $(300 \mathrm{~mL})$ as the packing solvent. All polymers were washed on-line with methanol-acetic acid $(9: 1, \mathrm{v} / \mathrm{v})$ mixture at a flow rate of $0.5 \mathrm{~mL} / \mathrm{min}$ until no further template 
bleed could be detected using HPLC.

\section{HPLC-analysis}

The HPLC system consisted of a JASCO PU-2080 chromatograph with a JASCO UV-2075 variable wavelength detector and a Rheodyne 7725 syringe loading sample $(20 \mu \mathrm{L})$ injector. For data analysis peak integration was performed using a Peak ABC Chromatography Workstation Ver.2.10 integrator. The apparatus provide assurance that all the UV-absorbing components are detected, if present in sufficient quantity. Analyses were run at flow-rates of $1.0 \mathrm{~mL} / \mathrm{min}$ with detection at $272 \mathrm{~nm}$. Capacity factors $\left(k^{\prime}\right)$ were determined from $k^{\prime}=\left(t-t_{0}\right) / t_{0}$, where $t$ is the retention time of a given species and $t_{0}$ is the elution time of the void marker (determined by injection of toluene). Effective separation factors $(\alpha)$ were calculated from the relationship $\alpha=K_{S P D}^{\prime} / K_{S Q X}^{\prime}$, where $K_{S P D}^{\prime}$ and $K_{S Q X}^{\prime}$ were the capacity factors of the SPD and SQX, respectively.

\section{Binding Experiment}

The concentration of standard solutions was $20 \mu \mathrm{mol} / \mathrm{L} \sim 1.0 \mu \mathrm{mol} / \mathrm{L}$ substrate (SPD and SQX). A calibration graph was found between concentration of subsrate and the absorbance. An accurately weighed $5 \mathrm{mg}$ portion of the polymer particles was transferred into a $50 \mathrm{~mL}$ centrifuge tube, $15 \mathrm{~mL}$ of vary concentration standard solution was added and the tube rotary. The SPD imprinted particles were equilibrated in the aqueous solution of SPD or SQX analogs at $4 \square$ for 12 hours. This solution was centrifuged at $2000 \mathrm{rpm}$ for 10 min at $4 \square$. The centrifugate was transferred into $50 \mathrm{~mL}$ volumetric flask. The absorbance of the solution was measured by HPLC with UV detector after the substrate bound. The concentration of substrate was estimated based on the standard curve. The value of substrate bound to the imprinted particle, [C] $(\mu \mathrm{mol} / \mathrm{g})$, was calculated according to the equation:

$$
[\mathrm{C}](\mu \mathrm{mol} / \mathrm{g})=\left(\mathrm{C}_{\mathrm{f}}-\mathrm{C}_{\mathrm{i}}\right) \mathrm{V} / \mathrm{g}
$$

where $\mathrm{C}_{\mathrm{f}}$ and $\mathrm{C}_{\mathrm{b}}$ were the molar concentrations $(\mu \mathrm{M})$ of SPD or other substrates after and before equilibration. $\mathrm{V}$ and $\mathrm{g}$ were volume of substrate solution and weight of imprinted particles, respectively.

\section{RESULTS AND DISCUSSION}

\section{Characterization of the SPD-imprinted polymer}

The SPD-MIP was prepared utilizing MAA as the functional monomer, which acts as both a hydrogen bond donor and acceptor with the imprint species. The bulk polymer was ground into powder, packed into HPLC columns and rendered it suitable for a stationary phase. The template was extracted from the polymer using $\mathrm{MeOH} / \mathrm{AcOH}(9: 1 ; \mathrm{v} / \mathrm{v})$ solution.

The solvent plays an important role in formation of the porous structure in MIP. It is also clear that the polarity of solvents used in the imprinting analyses affects the specificity of the polymers. Non-polar porogens such as toluene or polar porogen such as acetonitrile did not sufficiently dissolve SPD at low temperature. However, acetone is a non-protonic solvent that may not make the hydrogen bonding formation in the polymerization system. Thus, using acetone as the polymerization solvent is advantageous for the present imprinting, rebinding to the template. In this study, acetone is successfully used as solvent for preparing polymer-introduced recognition sites for SPD.

The results of this study show that better selectivity is obtained at the lower temperature ( $4 \square$ ) polymerization versus the identical polymers thermally (65 $\square$ ) polymerized. To polymerize at lower temperatures, it is necessary to use photochemical reaction (UV radiation). Figure 1 shows the binding results of SPD and SQX to MIP and non-MIP under different temperature polymerized. It was found that the value $[\mathrm{C}]_{\mathrm{SPD}}$ of SPD binding the imprinted particles decreased from $21.52 \mu \mathrm{mol} / \mathrm{g}$ to $14.96 \mu \mathrm{mol} / \mathrm{g}$, when the temperature of polymerization increased from $4 \square$ to $65 \square$. Meanwhile, the value $[\mathrm{C}]_{\mathrm{sox}}$ of SQX decreased from $15.12 \mu \mathrm{mol} / \mathrm{g}$ to $13.56 \mu \mathrm{mol} / \mathrm{g}$. It is clearly showed that the binding amounts of SPD to the imprinted particles were much higher than those of SQX. This indicated that in the MIP prepared with low temperature, the binding ability of SPD to the imprinted polymer increased because recognition site formation made homogeneity of the template in the particle. Therefore, the increased numbers of the imprinted sites led to the increased capacity of SPD binding when the temperature was $4 \square$ in the polymerization process. Furthermore, the binding amount of blank polymers (non-MIP) was clearly lower than that of MIP, showing that the absorptivities of blank polymer were physical adsorption (non-selective). Non-MIP lacked template molecules during polymerization, thus polymers would not be imprinted. Evidence of the binding results was the fact that this polymerization process was capable of imprinting the SPD shape into the polymer and at lower temperature was efficient to fix the shape of SPD template. In addition, the extraction manner left SPD imprinting sites in the polymer matrix. As a result, the recognized SPD was shown efficiently in the imprint particles. Overall, it can be seen that the MIP prepared in this research showed significant selectivity to the print molecule alone.

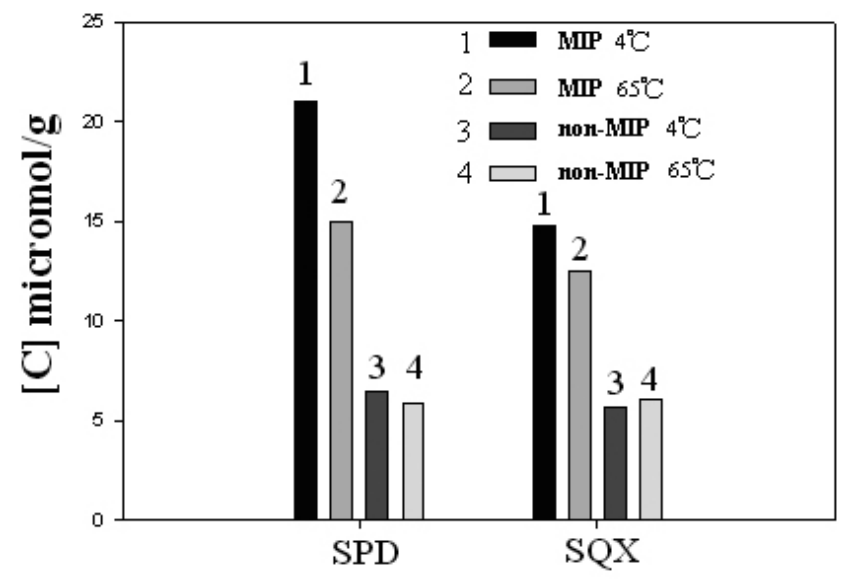

Figure1. Binding amounts of SPD and SQX to the imprinted particles. These particles prepared at $4 \square, 65 \square$ and used SPD as template.

Determination of binding parameters of the imprinted polymers

The Langmuir adsorption isotherm equation is one of the most widely used models to describe the equilibrium behaviors of adsorbate uptake. When an adsorbate (A) adsorbs to an adsorbent which contains specific binding sites (B). The isotherm models used in this work are the uni-Langmuir models ${ }^{23}$.

$$
\frac{1}{q}=\frac{1}{Q b c}+\frac{1}{Q}
$$

Where $q$ is adsorption capacity, $Q$ is the saturation capacities of sites on a heterogeneous surface, $b$ is the corresponding adsorption constants and $\mathrm{c}$ represents the concentration of adsorbate. It should be emphasized that $\mathrm{c}$ in Eq.(2) must be expressed as the molar concentration of adsorbate at equilibrium. Eq. (2) was used to fit the experimental data. Change in standard free energy of adsorption $\left(\square G^{O}\right)$ was determined by Van't Hoff equation at temperature T (K).

$$
\square G^{o}=-R T \ln K
$$

where $R$ is the universal gas constant $(8.314 \mathrm{~J} / \mathrm{mol} . \mathrm{K})$ and $K=q b$ is the thernlodynamic equilibrium constant. Test found a relationship between and - , namely, Langmuir plot showed in Figure 2. This figure obtained from the ioprinted polymer used SPD as template consists of two distinct straight lines with slop which gave dissociation constant. The maximum adsorption capacity $\left(Q_{\text {ma }}\right)$, equilibrium constant $(K)$ and standard free energy $\left(\square G^{O}\right)$ are summarized in Table 2. 


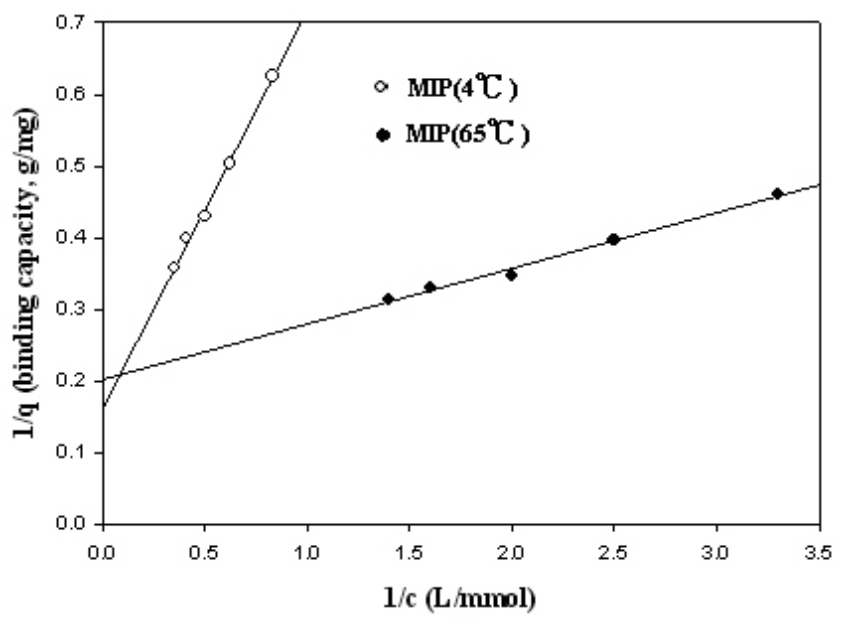

Figure2. Langmuir plots to estimate the binding parameters of SPD-MIP.

Table 2.- For SPD on the imprinted polymers. Fitted parameters equilibrium constant $\mathrm{K}$, the maximum adsorption capacity $(\mathrm{Q}$, and standard free energy $\left(\square \mathrm{G}^{\circ}\right)$ are calculated by using the Eq.(1) and (2).

\begin{tabular}{|c|c|c|c|}
\hline MIP system & $K(\mathrm{~L} / \mathrm{mol})$ & $Q_{\max }(\mu \mathrm{mol} / \mathrm{g})$ & $\begin{array}{c}\square G^{O}(\mathrm{~kJ} / \\
\mathrm{mol})\end{array}$ \\
\hline $4 \square$ & 5.55 & 22.30 & -4.24 \\
$65 \square$ & 4.54 & 18.23 & -3.74 \\
\hline
\end{tabular}

From the slope and intercept of the plot, the equilibrium constant $K$ and the apparent maximum number $Q$ of the higher affinity binding sites can be calculated to be $5.55 \mathrm{~L} / \mathrm{mol}$ and $22.30 \mu \mathrm{mol} / \mathrm{g}$ for MIP prepared at $4 \square$, respectively. In the same way, $K$ and $Q_{\text {of }}$ of the lower affinity bonding sites were calculated to be $4.54 \mathrm{~L} / \mathrm{mol}$ and $18.23 \mu \mathrm{mol} / \mathrm{g}$ for MIP prepared at 65 $\square$, respectively. Table 2 shows the values of $\square \mathrm{G}^{\circ}$ calculated from $K$ by using Eq.(3), indicating that SPD interacts most strongly with the polymers. Therefore, the MIP synthesized with MAA is expected to give the highest selectivity to SPD. As described, above we can estimate the selectivity of MIPs by recovery measurements of an analyte and other structurally related analogues.

Molecular recognition of SPD and SQX with molecularly imprinted polymer as the stationary phase of HPLC

In single or binary substrate solution, $1 \mathrm{~g} / \mathrm{L}$ of SPD and SQX solution or mixture of these two compounds was injected for analysis in a total volume of $20 \mu \mathrm{L}$ and eluted isocratically at a flow-rate of $1.0 \mathrm{~mL} / \mathrm{min}$. Subsequently, the imprinting effect was evaluated by HPLC with UV detection. The results were shown in Figure 3. Molecular recognition effect of SPD from the binary mixture solution was evaluated with separation factor as described in experimental section. Validation revealed that the retention time of SPD and SQX were 7.96 min and $3.63 \mathrm{~min}$ when the flow rate was $1 \mathrm{~mL} / \mathrm{min}$.
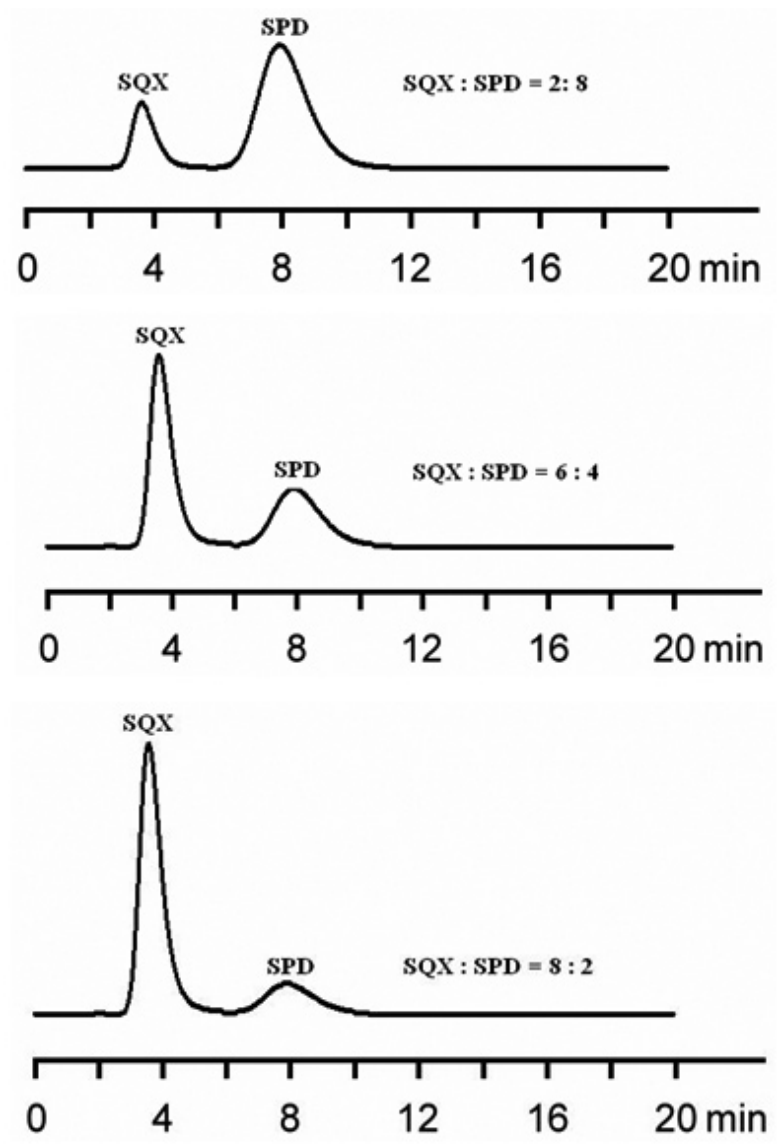

Figure 3.- Chromatograms at $272 \mathrm{~nm}$ of SPD and SQX by HPLC using molecularly imprinted polymer as the stationary phase.

Table 3 shows the retention time, retention factor and separation factor $(\alpha)$ values obtained after separation by HPLC. The SPD and SQX are readily and rapidly separated when $34 \mathrm{mmol} / \mathrm{mL} \mathrm{NaH}_{2} \mathrm{PO}_{4}$ buffer solution/ acetonitrile $(3 \square 2, \mathrm{v} / \mathrm{v})$ is used as the mobile phase. From Table 3, the retention times for SPD and SQX were 7.77 7.95 $\mathrm{min}$ and $3.55 \sim 3.64 \mathrm{~min}$, respectively. The difference of retention time $\left(\square \mathrm{t}_{\mathrm{R}}\right)$ between SPD and SQX were 4.14 4.41 min. The chromatographic run was completed in less than $10 \mathrm{~min}$ and completely separated from the other peaks under a flow rate of $1 \mathrm{~mL} / \mathrm{min}$. It was found that the values of $\alpha$ obtained for the imprinted particles prepared at $4 \square$ were 2.55 2.71 for SPD/SQX, which was a nearly constant. The SPD-imprinted polymer prepared at $4 \square$ showed higher selectivity to SPD than that of $65 \square$, further evaluation was carried in binary mixture with a total of $1 \mathrm{~g} / \mathrm{L}$.

The retention time for SPD and SQX were $0.96 \mathrm{~min}$ and $1.02 \mathrm{~min}$ when used blank polymer (non-MIP) as the stationary phase, and the difference of retention time $\left(\square \mathrm{t}_{\mathrm{R}}\right)$ between SPD and SQX was $0.06 \mathrm{~min}$. It can be seen that the blank polymer had little selectivity for SPD and SQX, while the retentivity and selectivity of the imprinted polymer for the two molecules were greatly strengthened by molecular imprinting as indicated by the imprinted particles. The good imprinting effect was likely due to the carboxylic group on the monomer and the electrostatic interaction or hydrogen bonding between imprinted polymer and template molecules in the polymerization. Apparently, the SPD-imprinted particles selectively bound SPD rather than SQX. Although SQX was structurally close to the SPD template, the retentions of SPD were higher than SQX on the imprinted polymers. These results indicated that imprinted polymers formed recognition sites for the SPD compound as the template molecules. The significant difference in the retention was due to the fact that SPD molecules were removed by washing the polymer matrix with an acetic acid/methanol solution, and therefore the polymers left cavities of a complementary size and shape, providing a great deal of free amino residue groups in the copolymer matrix. The higher population of free amino residue group in the polymer particles seems to increase the affinity of the solutes. The 
reason is that the removal of SPD leaves a clear cavity whose morphology is similar to SPD on the particles. Thus the stationary phase utilized the cavity and the free amino residue groups caught SPD. Therefore, these data strongly suggested that the SPD imprinted polymer had effective selectivity by hydrogen bonding to separately bind in the binary components.

Table 3.- Composition of solution with a total of $1 \mathrm{~g} / \mathrm{L}$ and resolution of SPD and SQX used MIP as the stationary phase of HPLC.

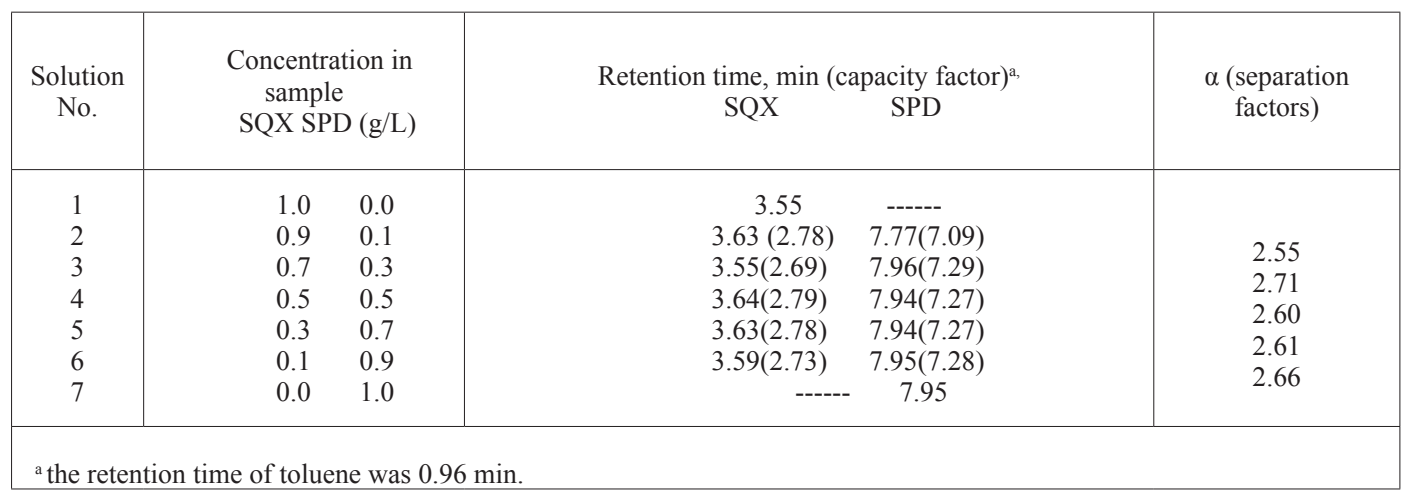

\section{CONCLUSION}

SPD-imprinted polymers were successfully prepared by bulk polymerization at $4 \square$ in acetone solvent. It was efficient to fix the shape of SPD template into the particle through hydrogen bonding with low temperature. However, under high temperature treatment it showed lower effect on imprinting. Results showed that SPD imprinted particle with $4 \square$ preparation provided high recognition and selectivity to SPD rather than SQX. The SPD-imprinted particle had effective separation property of the binary components. Therefore, it was meaningful to develop SPD recognition materials applying in the various fields such as chromatographic separation, biosensors and drug therapy.

\section{ACKNOWLEDGEMENTS}

We thank Mingdao University for financial support.

\section{REFERENCES}

1. Kishida, K. ; Furusawa, N. J. Chromatogr. A 937, 49, (2001).

2. Berzas Nevado, J. J.; Castañeda Peñalvo, G..; Guzma'n Bernardo, F-J. J. Chromatogr. A 870, 169, (2000).

3. Furusawa, N. Anal. Chim. Acta 481, 255, (2003).

4. Ito, Y.; Oka, H.; Ikai, Y.; Matsumoto, H.; Miyazaki, Y.; Nagase, H. $J$. Chromatogr. A 898, 95, (2000).

5. Gehring, T. A.; Griffin, B.; Williams, R.; Geiseker, C.; Rushing, L. G..; Siitonen, P. H. J. Chromatogr. B 840, 132, (2006).
6. Stoev, G..; Michailova, A . J. Chromatogr. A 871, 37, (2000).

7. Titus, A . M.; Msagati ,M. M. N. Talanta 64, 87, (2004).

8. Li, H.; Kijak, P. J.; Turnipseed, S. B.; Cui, W. J. Chromatogr. B 836, 22, (2006).

9. Garc'ia , I.; Ortiz, M. C.; Sarabia, L.; Aldama, J. M. Anal. Chimi. Acta $\mathbf{5 8 7}, 222$, (2007).

10. Norrlo,"W. O.; Glad, M.; Mosbach, K. J. Chromatogr. 299, 29, (1984)

11. Arshady, R.; Mosbach, K. Makromol. Chem. 182, 687, (1981).

12. Wulff, G..; Schauhoff, S. J. Org. Chem. 56, 395, (1991).

13. Wulff, G..; Vesper, R. J. Chromatogr. 167,171, (1978).

14. Hwang,C .C .; Lee, W C. J. Chromatogr. A 962, 69 ,(2002).

15. Patel, A.; Fouace, S.; Steinke, J.H.G.. Anal. Chim. Acta. 504, 53, (2004).

16. Petcu, M.; Schaare, P. N.; Cook, C. J. Anal. Chim. Acta 504, 73, (2004).

17. Klein, J. U.; Whitcombe, M. J.; Mulholland, F.; Vulfson, E.N. Angew. Chem., Int. Ed. 38, 2057, (1999).

18. Fairhurst, R. E.; Chassaing, C.; Venn, R. F.; Mayes, A G.. Biosens. Bioelectron. 20, 1098, (2004).

19. Dirion, B.; Lanza, F.; Sellergren, B.; Chassaing, C.; Venn, R.; Berggren, C. Chromatographia. 56, 237, (2002).

20. Ferrer, I.; Lanza, F.; Tolokan, A.; Horvath, V.; Sellergren, B.; Horvai, G..; Barcelo, D. Anal. Chem. 72, 3934, (2000).

21. Kanekiyo, Y.; Naganawa, R.; Tao, H. Chem. Commun. 2698, (2002).

22. Hung, C.Y.; Hwang, C.C. J. Chromatogr. Sci. 46, (2008)

23. Hammes, G.. G.. Thermodynamics and Kinetics for the Biological Sciences, Wiley-Interscience, New-York (2000). 